\title{
Determinants of uptake of whole-body skin self-examination in older men
}

Josephine Auster, MAppSc ${ }^{(1)}$; Cameron Hurst ${ }^{(1)}$; PhD; Rachel E Neale, $\mathrm{PhD}^{(2)}$; Phillipa Youl, $\mathrm{MPh}^{(3)}$; David C Whiteman, $\mathrm{PhD}^{(2)}$; Peter Baade, $\mathrm{PhD}^{(3)}$; Monika Janda, $\mathrm{PhD}^{(1)}$

1. School of Public Health, Institute of Health and Biomedical Innovation, Queensland University of Technology, Queensland, Australia

2. Queensland Institute of Medical Research, Queensland, Australia

3. Viertel Centre for Research in Cancer Control, Cancer Council Queensland, Queensland, Australia

\section{Corresponding author:}

Monika Janda, $\mathrm{PhD}$

Senior research fellow

Queensland University of Technology

Victoria Park Rd

Kelvin Grove, Brisbane, 509

Australia

Phone: 0061731383018

Fax: 0061731383130

Email: m.janda@qut.edu.au 


\title{
Determinants of uptake of whole-body skin self-examination in older men
}

\begin{abstract}
Early detection through whole-body Skin Self-Examination (wbSSE) may decrease mortality from melanoma. Using the Health Action Process Approach (HAPA) or Health Belief Model (HBM) we aimed to assess determinants of uptake of wbSSE in 410 men 50 years of older who participated in the control group of a randomised trial. Overall, the HAPA was a significantly better predictor of wbSSE compared to the HBM ( $<<0.001)$. The construct of self-efficacy in the HBM was a significant predictor of future wbSSE $(p=0.001)$, while neither perceived threat $(p=0.584)$ nor outcome expectations ( $p=0.220$ ) were. In contrast, self-efficacy, perceived threat, and outcome expectations predicted intention to perform SSE, which predicted behaviour $(\mathrm{p}=0.015)$. The HAPA construct volitional self-efficacy was also associated with wbSSE $(p=0.046)$. The use of the HAPA model for future SSE interventions for this population is warranted.
\end{abstract}

Keywords: melanoma; self-examination; early diagnosis; middle aged males; psychological theories; 


\section{Introduction}

Australia has the highest incidence of skin cancer worldwide ${ }^{1}$, and men aged 50 years or older have been identified as a high risk group for melanoma ${ }^{2}$. Secondary skin cancer prevention measures, such as skin self-examination (SSE), have the potential to improve survival from melanoma ${ }^{3}$. While there is no longitudinal evidence for the effectiveness of SSE, it may reduce mortality from melanoma by up to $63 \%^{4}$, as well as reducing the thickness and severity of melanoma at diagnosis ${ }^{4-7}$. Despite this, reported prevalence of SSE in the general population is low, especially in older men ${ }^{8}$, and men are less likely to engage in preventative SSE than women ${ }^{9}$.

Interventions to increase preventative behaviours such as SSE are often based on theoretical models ${ }^{10}$. The Health Belief Model (HBM) in particular, is the most widely applied model of health behaviour ${ }^{11}$ and many scholars believe that it forms the foundations for behaviour change practice ${ }^{10}$. It rests on the premise that an individual's likelihood of performing a behaviour is predicted by their underlying beliefs about that behaviour and the health problem it will prevent ${ }^{12}$. The major constructs of the HBM are a person's perceived threat (perceived seriousness and perceived susceptibility) from a particular health problem, their expected outcomes (benefits and barriers) from performing the preventative behaviour and self-efficacy, which is belief in their ability to correctly perform the behaviour ${ }^{11,13}$. Although there is evidence that the HBM effectively predicts health behaviours ${ }^{14,15}$, it has some limitations ${ }^{11,14,16}$. It has been argued that the model has poorly defined constructs ${ }^{11}$ and focuses on motivational factors without considering how intention to perform a behaviour translates into actual uptake and long-term maintenance of that behaviour. For example, while the HBM indicates that intervening on a person's motivation to wbSSE will increase their intention to perform the behaviour, it gives no indication of how likely it is that this will lead to actual uptake of wbSSE. 
By contrast, the Health Action Process Approach (HAPA) is a more recently developed model ${ }^{17,18}$ that attempts to overcome weaknesses of earlier social cognitive theories by including constructs which stand between intention and behaviour, thereby addressing the 'intention-behaviour' gap ${ }^{17}$. There are two implicit phases in the model: the motivational phase, which deals with motivational constructs leading to the formation of an intention to change behaviour; and the volitional phase, which specifies constructs that explain variance in actual behaviour change ${ }^{17}$ (see Figure 1).

\section{Insert Figure 1 about here}

The motivational phase is defined by three constructs, which are similar to those specified in the HBM. Action self-efficacy refers to an individual's belief in their ability to correctly perform a behaviour, while risk perception is a measure of a person's perceived vulnerability to develop skin cancer (susceptibility) and severity the person attaches to such diagnosis ${ }^{19}$. Outcome expectancies are a person's positive and negative expectations about the consequences of taking up a behaviour ${ }^{19}$. These constructs are hypothesised to predict intention to perform a behaviour. In the volitional stage are the constructs of action planning and volitional self-efficacy. Action planning involves a person making plans to perform a behaviour, such as when, where and how they will do it ${ }^{17}$. Volitional self-efficacy refers to a person's belief in their capacity to overcome unexpected barriers that affect their ability to maintain that behaviour, and belief in their ability to overcome setbacks or relapses into old behaviour ${ }^{17}$.

A review of HAPA’s efficacy presented empirical evidence for the predictive value of the model ${ }^{17}$. In five studies HAPA constructs explained significant amounts of variance for differing kinds of behaviours, and the hypothesised pathways between constructs were 
confirmed. For example, in a study measuring physical activity in cardiac rehabilitation patients, volitional constructs explained 32\% of variation in physical activity behaviour ${ }^{20}$.

Within the current context of SSE, numerous studies have cited either health behaviour models or a combination of constructs from multiple models to guide the development of SSE interventions ${ }^{21-25}$ Despite this, neither the complete HBM nor HAPA models have been specifically tested for their predictive value for SSE in any population. Given criticisms of the HBM and potential advantages of the HAPA, it may be that the HAPA provides a better theoretical basis for why some men choose to take up and maintain wbSSE, and others do not. If the HAPA explains SSE behaviour more completely then the HBM, the constructs it includes may provide a potential focus for future interventions. Using the HAPA or HBM models, the aim of the present study therefore was to assess determinants of uptake of wbSSE behaviour in these men 50 years or older. 


\section{Methods}

Ethical considerations. Ethical clearance was granted from the Queensland University of Technology (QUT) Human Research Ethics Committee, and the trial was registered with the Australian New Zealand Clinical Trials Registry (ANZCTR N12608000384358).

Randomised trial. This analysis used data from a randomised intervention trial to improve wbSSE, the Skin Awareness Study. Full details of the study design and methods have been previously described ${ }^{21,26}$. In brief, the Skin Awareness Study was conducted between 2008 and 2010 in Queensland, Australia. Men aged 50 years or older randomly selected from the Australian electoral roll (enrolling to vote is compulsory in Australia) were mailed an invitation to participate. Of the total 2276 (78\%) men who responded to the initial mail-out, 1019 (45\%) refused participation and 289 (12.7\%) were deemed ineligible due to cognitive or hearing impairment, non-English speaking ability, no access to either a DVD or VCR player (required for the intervention) or a previous melanoma diagnosis. Overall, 930 eligible men agreed to participate and were randomised to either the intervention group or the control group. Two weeks following a baseline measure, the intervention group received a tailored video-delivered intervention, while the control group received 'usual care' print-based materials. Data were collected by Computer Assisted Telephone Interviews (CATIs) at baseline, and again at 7 and 13 months after participants received the study materials.

Current analysis. To reduce variation induced by the intervention, only data from control group participants were used for the analyses described here. Furthermore, as data relating to the HBM and HAPA were only collected at baseline and the 13-month time point, data from the 7-month time point was not included in the analysis. Participants were included if they had completed interviews at both the baseline and 13-month time points. Of those who 
completed the baseline interview $(n=460), 50$ did not complete the 13-month interview, leaving a final sample of 410. Participants who did not complete both interviews were similar to those who did in terms of basic demographic characteristics.

Measures. Data on demographic and health characteristics including area of residence, marital status, age group, education, income, and past participation in other cancer screening tests were collected at baseline.

The primary outcome variable used for these analyses was wbSSE at 13 months. Men were classified as having performed a wbSSE in the last 6 months if they had indicated that they had examined each of 13 body areas and that they had either had a person help them or used mirrors to check hard to see parts of the body.

Measures were selected from the baseline interview which best represented the similar HBM and HAPA constructs of self-efficacy ('action self-efficacy' in the HAPA), perceived threat, and outcome expectations ('outcome expectancies' in the HAPA; Table 2). Measures of the remaining HAPA constructs were selected from either the baseline or 13month interview in line with Schwarzer's ${ }^{19}$ suggestion that 'at least two measurement points are recommended to test the model assumptions: [perceived threat], [outcome expectations], action self-efficacy, and intention at Wave 1, and planning, maintenance self-efficacy, recovery self-efficacy, and behaviour at Wave 2'. Several of the measures used were taken from previous SSE research ${ }^{8}$; while others were developed for the purposes of this study.

Statistical analysis. Basic descriptive statistics were generated to describe participants' demographic and health characteristics.

Structural Equation Modelling (SEM) with Mplus version $6^{27}$ was used to test hypothesised associations between model constructs and to compare the two models. A 
maximum likelihood (ML) estimation and logistic regression were used to estimate regression coefficients (unstandardised) and significance values for each parameter. We considered relationships between constructs to be important if they were statistically significant at the standard $\mathrm{p}<0.05$ level.

The models were compared using a nested comparison based on the loglikelihood ratio test ${ }^{19}$ and Akaike's information criterion (AIC) was reported for each model. This statistic provides an indication of model fit, adjusted for parsimony. As it is not normed on a 0-1 scale, AIC can only be used in a comparative sense; there is no objective cut-off point which indicates a 'good fit'. When comparing two models, however, smaller values indicate a more well fitting and parsimonious model ${ }^{28}$.

Variables. SEM allows for the inclusion of latent variables in a model, which represent an underlying construct measured by multiple data items. In the present study, multiple data were available for a single construct only: self-efficacy at baseline (three items as listed in Table 2). To determine whether these items appropriately measured the underlying construct, a single factor measurement model was specified and estimated using the maximum likelihood method. To identify the model, error variances for 'correct SSE' and 'monthly SSE' were constrained to be equal, as they were found to be similar in an initial model. The model fit was satisfactory: $\chi^{2}=0.847$ ( $\mathrm{df} 1, \mathrm{p}=0.358$ ); Root Mean Square Error of Approximation $($ RMSEA) $<0.001$; Comparative Fit Index $(\mathrm{CFI})=0.999$. Standardised factor loadings were all significant at the $\mathrm{p}<0.001$ level (see Fig 2).

The remaining variables were directly derived from single or combined items as listed in Table 1. The constructs of planning, intention and wbSSE were specified as categorical variables. 
Model specification. Model 1 (Fig 2) is a simple representation of the HBM, where constructs on the left (self-efficacy, perceived threat, and outcome expectations as measured at baseline), were specified as predictors of wbSSE at 13 months.

Insert Figure 2 about here

Extra constructs were added to Model 1 to represent the hypothesised pathways of the HAPA (Fig 3).

\section{Insert Figure 3 about here}

Given Schwarzer's hypothesis concerning the closure of the 'motivational' phase in forming intentions to perform a behaviour ${ }^{19}$, intention was included as a mediator between the constructs that motivate behaviour performance and wbSSE. In addition, the theoretical framework of the HAPA model suggests that an individual must make plans for behaviour performance prior to executing it ${ }^{19}$. Given this, the construct of SSE planning, as measured at 13 months, was specified as a mediator between intention at baseline and wbSSE at 13 months. Schwarzer also reported that volitional self-efficacy is important in maintaining behaviour change and overcoming obstacles and relapses over time ${ }^{19}$. This construct, also measured at 13 months, was added into the model as a predictor of wbSSE.

In order to perform a nested comparison of the HBM with the HAPA, model 2 was run again, constraining the paths to HAPA constructs to equal zero (Figure 4).

\section{Insert Figure 4 about here}

Whether the HAPA model was significantly better than the HBM at predicting SSE behaviour was tested using a likelihood ratio test (80), for which the formula is as follows:

$D=-2 \times($ likelihood for null model $)-\ln ($ likelihood for alternative model $) \square$ 
Degrees of freedom are calculated by subtracting the number of free parameters for the null model from the number of free parameters from the alternative model. If the difference statistic D was found to be significant, the HAPA was considered a significantly better model for predicting wbSSE behaviour.

\section{Results}

Participants’ baseline demographic and health characteristics are described in Table 1. The majority of participants were married or living with a partner (85.6\%); and most (80.7\%) were aged between 50 and 70 years. In addition, most participants indicated they had previously participated in other cancer screening tests; namely Prostate Specific Antigen (PSA) tests (78.8\%) and Fecal Occult Blood Tests (FOBT) (58.3\%). $\underline{\text { A full description of }}$ baseline characteristics of the control group has been published elsewhere ${ }^{21} . \underline{\text { In multivariable }}$ analyses using the whole study sample, demographic characteristics were not found to be significantly associated with wbSSE ${ }^{21}$.

Figures 2 and 3 demonstrate the results of each analysis. In all models, unstandardised regression coefficients are displayed for each of the paths, with odds ratios shown in brackets where they could be calculated (for categorical variables).

Model 1 represents the HBM. In this model, the baseline measure of action selfefficacy was found to significantly predict wbSSE at 13 months $(\beta=0.316 ; p=0.001)$; while neither perceived threat $(\beta=0.055 ; p=0.584)$ nor outcome expectations $(\beta=0.244 ; p=0.220)$ significantly predicted wbSSE. For this model AIC $=6372.80$.

In Model 2 (representing the HAPA), volitional self-efficacy was a significant predictor of wbSSE $(\beta=0.383, p=0.046)$ but SSE planning did not significantly predict $\operatorname{wbSSE}(\beta=0.169, \mathrm{p}=0.085)$. However, the direct path from intention to wbSSE (not shown in 
the diagram) remained significant $(\beta=1.11 ;$ OR: $2.21 ; \mathrm{p}=0.015)$. For this model AIC $=$ 8687.60.

In addition to the individual models 1 and 2 above, we performed a nested comparison of the HBM and HAPA models, first restricting the HAPA paths to zero thus only allowing HBM components to contribute, then allowing and unconstrained model with all HAHA paths estimated (Table 3). Based on loglikelihood comparisons (Table 3), the HAPA fitted the data significantly better (chi-square $=164.34 ; \mathrm{df}=8, \mathrm{p}<0.001$ ), even taking into account the additional complexity it contained. This is further demonstrated by a comparison of the AIC statistics; the HAPA model had a lower AIC (AIC $=8688.85$ compared to the HBM-HAPA restricted model AIC=8837.18), indicating a comparatively better model.

\section{Comment}

A Structural Equation Modelling analysis of data from the Skin Awareness Study provided preliminary evidence that the HAPA model is better at predicting wbSSE in a sample of older men than the HBM. Furthermore, the findings tend to support the hypothesised pathways of the HAPA model.

Previous analysis of a similar sample revealed no significant associations between wbSSE and demographic characteristics $^{21}$._Analysis of the HBM revealed that only selfefficacy at baseline was associated with reported wbSSE at 13 months, while neither outcome expectations nor perceived threat predicted wbSSE. This differs from the findings of previous cross-sectional studies, which measured items representing HBM constructs. Perceived threat for melanoma was found to increase the odds of having performed SSE in three studies $8,29,30$. These studies included samples from the general population and younger adults. Threat perception may increase preventative behaviours more in these populations compared to 
older men, a group who have been shown to have a lower uptake of $\mathrm{SSE}^{8,9}$. In terms of outcome expectations, a study involving relatives of melanoma survivors found that barriers to performing SSE decreased the odds of having performed the behaviour in the past, and perceived benefits of SSE increased the odds ${ }^{31}$. Similar correlations may have been overlooked in the current study, as benefits and barriers were not measured as individual constructs. However, in addition to these factors, results of the present study may also differ due to its longitudinal study design. While the cross-sectional studies mentioned above demonstrated associations between HBM constructs and past SSE behaviour, in this study only self-efficacy, and not perceived threat or outcome expectations, prospectively predicted future wbSSE. This raises the possibility that the HBM may not be a sufficient model to predict the range of variance in future wbSSE behaviour in this population.

The HAPA model was found to fit the data better than the HBM, and all but one of the hypothesised pathways were significant. Firstly, all three motivational variables were significantly associated with intention to perform SSE at baseline, which in turn increased the odds of having performed wbSSE at 13 months. Although no previous longitudinal work has linked intention to perform SSE with the behaviour, these findings correspond with the body of evidence from other areas of health research demonstrating that intentions are usually associated with performing a health behaviour ${ }^{32}$. Secondly, while planning slightly mediated the effect of intention on wbSSE, it did not significantly increase the odds of wbSSE when volitional self-efficacy was also included in the model. Planning has been linked to other health behaviours in previous studies using the HAPA model, but this has not been assessed in the context of SSE. For example, in a sample of 418 women, planning was the best direct predictor of breast self-examination ${ }^{33}$; and similarly it was significantly associated with seatbelt use in a population of adolescents ${ }^{34}$. In a study of dietary behaviours in a Korean population, planning predicted behaviour in women but not in men, while self-efficacy was 
of equal predictive value in both genders ${ }^{35}$. In the current population of men aged 50 years or older, the results indicate that self-efficacy may be a more important proximal predictor of wbSSE than planning. Volitional self-efficacy at 13 months (confidence in ability to maintain the behaviour and recover from set-backs) was significantly associated with wbSSE, and was predicted by action self-efficacy at baseline (having confidence in ability to take up SSE).

In regards to practice, these findings could be applied by designing future interventions towards variables more proximal to behaviour (planning and volitional selfefficacy). This could be particularly relevant if, as Schwarzer suggests, different parts of the model are relevant for people in different mindsets ${ }^{17}$. According to the stage version of the HAPA model, people can be divided into three groups: non-intenders (for example, do not intend to perform wbSSE); intenders (intend to perform wbSSE but have not yet done so); and actors (are already performing wbSSE) ${ }^{17}$. Some past studies in other health behaviour fields targeting constructs more proximal to behaviour have led to increases in those behaviours ${ }^{17}$. For example, an intervention to improve action planning in patients already motivated to increase their physical activity ('intenders') successfully increased levels of activity $^{36}$. Another study increased the initiation and/or maintenance of breast selfexamination via an intervention focused on action and maintenance self-efficacy ${ }^{37}$.

Limitations. Limitations of the Skin Awareness Study have been published elsewhere ${ }^{21}$. Briefly, all data were collected via self-report, introducing potential recall bias. Men who participated in the study may have had higher incomes and been more health conscious than the general population, making uptake of wbSSE more likely ${ }^{26}$. Furthermore, specific limitations exist that are unique to the particular analyses used here. Firstly, the single items used in this study were not specifically designed to measure constructs of the HBM or HAPA and therefore may not reflect them accurately. For the HBM, as no item was available for 
perceived severity, we used combined measures of perceived threat (perceived susceptibility as a proxy) and outcome expectations (composite score of perceived barriers/perceived benefits). For the HAPA, while the measures used in this analysis for perceived threat, selfefficacy, intentions, and planning were similar to recommended items, the measure for the construct of outcome expectancies was quite different ${ }^{19}$. A composite score from data on a single negative outcome item was used ('I think checking my skin would make me anxious'), which may not have encompassed the range of negative outcome expectations about SSE; and two positive outcome items: 'Checking my skin regularly is a priority for me' and 'It is important to check my skin for skin cancer even if I have no symptoms', which were assumed to be sufficient proxies.

Finally, as this study focused on constructs related to two specific models, it did not consider the influence of all factors potentially relevant to the uptake of wbSSE; for example, the presence of psychological disorders, which may affect adherence to health promoting $\underline{\text { behaviours }}^{38}$. Future research could investigate the affect of these and other relevant factors in conjunction with a model such as HAPA.

Conclusions. In summary, men aged 50 years or older are at high risk of melanoma, and are less likely to perform wbSSE than the general population. A strength of the current study was its longitudinal design, and the results provide preliminary support for the hypothesis that the HAPA model is better at predicting wbSSE in this context. However, due to limitations in data measurement, further investigation is required to confirm this. There is also some indication that intervening on motivational variables alone may increase intention to examine skin, but not necessarily the behaviour itself. Future interventions for this high-risk population could therefore benefit from considering variables more proximal to actual 
behaviour (such as volitional self-efficacy and action planning) as described in the HAPA model. 


\section{References}

1. Curado M, Edwards B, Shin H, et al. Cancer Incidence in Five Continents. Lyon: International Agency for Research on Cancer; 2007.

2. Queensland Cancer Registry \& Queensland Cancer Fund. Cancer in Queensland: Incidence and Mortality, 1982 to 2004. Spring Hill: Queensland Cancer Fund; 2007.

3. Friedman RJ, Rigel DS, Kopf AW. Early detection of malignant melanoma: The role of physician examination and self-examination of the skin. CA: A Cancer Journal for Clinicians. 1985;35(3):130-151.

4. Berwick M, Begg CB, Fine JA, Roush GC, Barnhill RL. Screening for cutaneous melanoma by skin self-examination. J Natl Cancer Inst. 1996;88(1):17-23.

5. Carli P, De Giorgi V, Palli D, et al. Dermatologist detection and skin self-examination are associated with thinner melanomas: results from a survey of the Italian Multidisciplinary Group on Melanoma. Arch Dermatol. 2003;139(5):607-612.

6. Pollitt RA, Geller AC, Brooks DR, Johnson TM, Park ER, Swetter SM. Efficacy of skin self-examination practices for early melanoma detection. Cancer Epidemiol Biomarkers Prev. 2009;18(11):3018-3023.

7. Terushkin V, Halpern AC. Melanoma early detection. Hematol Oncol Clin North Am. 2009;23(3):481-500.

8. Aitken JF, Janda M, Lowe JB, et al. Prevalence of whole-body skin self-examination in a population at high risk for skin cancer (Australia). Cancer Causes Control. 2004;15(5):453-463.

9. Kasparian NA, McLoone JK, Meiser B. Skin cancer-related prevention and screening behaviors: a review of the literature. J Behav Med. 2009;32(5):406-428. 
10. Glanz K, Rimer BK, Su SM. Theory at a Glance: A Guide for Health Promotion Practice. Available at: http://www.cancer.gov/PDF/481f5d53-63df-41bc-bfaf5aa48ee1da4d/TAAG3.pdf. Accessed September 10, 2010.

11. Strecher VJ, Rosenstock IM. The Health Belief Model. In: Glanz K, Lewis FM, Rimer BK, eds. Health Behavior and Health Education. San Francisco: Jossy-Bass Publishers; 1997:41-59.

12. Hayden J. Introduction to Health Behavior Theory. Sudbury, MA: Jones and Bartlett Publishers; 2009.

13. Rosenstock IM, Strecher VJ, Becker MH. Social Learning Theory and the Health Belief Model. Health Education Quarterly. 1988;15(2):175-183.

14. Harrison JA, Mullen PD, Green LW. A meta-analysis of studies of the Health Belief Model with adults. Health Edu Research. 1992;7(1):107-116.

15. Janz NK, Becker MH. The Health Belief Model: a decade later. Health Education Quarterly. 1984;11:1-47.

16. Armitage CJ, Conner M. Social cognition models and health behaviour: A structured review. Psychology \& Health. 2000;15(2):173-189.

17. Schwarzer R. Modeling health behavior change: How to predict and modify the adoption and maintenance of health behaviors. Applied Psychology-an International Review-Psychologie Appliquee-Revue Internationale. 2008;57(1):1-29.

18. Sutton S. How does the health action process approach (HAPA) bridge the intentionbehavior gap? An examination of the model's causal structure. Applied Psychology-an International Review-Psychologie Appliquee-Revue Internationale. 2008;57(1):66-74.

19. Schwarzer R, Sniehotta FF, Lippke S, et al. On the Assessment and Analysis of Variables in the Health Action Process Approach: Conducting an Investigation. 
Available at: http://web.fu-berlin.de/gesund/hapa_web.pdf. Accessed September 3, 2010.

20. Sniehotta FF, Scholz U, Schwarzer R. Bridging the intention-behaviour gap: Planning, self-efficacy, and action control in the adoption and maintenance of physical exercise. Psychology \& Health. 2005;20(2):143 - 160.

21. Janda M, Baade PD, Youl PH, et al. The skin awareness study: promoting thorough skin self-examination for skin cancer among men 50 years or older. Contemp Clin Trials. 2010;31(1):119-130.

22. Oliveria SA, Chau D, Christos PJ, Charles CA, Mushlin AI, Halpern AC. Diagnostic accuracy of patients in performing skin self-examination and the impact of photography. Arch Dermatol. 2004;140(1):57-62.

23. Robinson JK, Turrisi R, Mallett K, Stapleton J, Pion M. Comparing the Efficacy of an In-Person Intervention With a Skin Self-examination Workbook. Arch Dermatol. 2010;146(1):91-94.

24. Robinson JK, Turrisi R, Stapleton J. Efficacy of a partner assistance intervention designed to increase skin self-examination performance. Arch Dermatol. 2007;143(1):37-41.

25. Weinstein ND. Misleading tests of health behavior theories. Ann Behav Med. 2007;33(1):1-10.

26. Janda M, Neale RE, Youl P, Whiteman DC, Gordon L, Baade PD. Impact of a videobased intervention to improve the prevalence of skin self-examination in men 50 years or older: the randomized skin awareness trial. Arch Dermatol. 2011;147(7):799-806.

27. Muthen \& Muthen. Mplus version 6.1998-2010.

28. Tabachnick BG, Fidell LS. Using Multivariate Statistics. 5th ed. Boston: Pearson/Allyn \& Bacon; 2007. 
29. Douglass HM, McGee R, Williams S. Are young adults checking their skin for melanoma? Aust N Z J Public Health. 1998;22(5):562-567.

30. Robinson JK, Rigel DS, Amonette RA. What promotes skin self-examination? J Am Acad Dermatol. 1998;38(5):752-757.

31. Manne S, Fasanella N, Connors J, Floyd B, Wang H, Lessin S. Sun protection and skin surveillance practices among relatives of patients with malignant melanoma: prevalence and predictors. Prev Med. 2004;39(1):36-47.

32. Sheeran P. Intention-Behavior Relations: A Conceptual and Empirical Review. European Review of Social Psychology. 2002;12:1 - 36.

33. Luszczynska A, Schwarzer R. Planning and Self-Efficacy in the Adoption and Maintenance of Breast Self-Examination: A Longitudinal Study on Self-Regulatory Cognitions. Psychology \& Health. 2003;18(1):93 - 108.

34. Schwarzer R, Schüz B, Ziegelmann J, Lippke S, Luszczynska A, Scholz U. Adoption and maintenance of four health behaviors: Theory-guided longitudinal studies on dental flossing, seat belt use, dietary behavior, and physical activity. Ann of Behav Med. 2007;33(2):156-166.

35. Renner B, Kwon S, Yang B-H, et al. Social-cognitive predictors of dietary behaviors in South Korean men and women. In J Behav Med. 2008;15(1):4-13.

36. Sniehotta FF, Scholz U, Schwarzer R. Action plans and coping plans for physical exercise: A longitudinal intervention study in cardiac rehabilitation. British Journal of Health Psychology. 2006;11:23-37.

37. Luszczynska A. Change in breast self-examination behavior: Effects of intervention on enhancing self-efficacy. In J Behav Med. 2004;11(2):95-103. 
38. Pompili M, Serafini G, Del Casale A, et al. Improving adherence in mood disorders: the struggle against relapse, recurrence and suicide risk. Expert Review of Neurotherapeutics. 2009;9(7):985-1004. 


\section{Figure Key}

Figure 1: Health Action Process Approach (HAPA) models

Figure 2: Model 1. Prediction of behaviour - Health Belief Model

Figure 3: Model 2. Prediction of intention and behaviour including planning and volitional self-efficacy

Figure 4: Model 3. Null model with HAPA paths constrained 
Figure 1

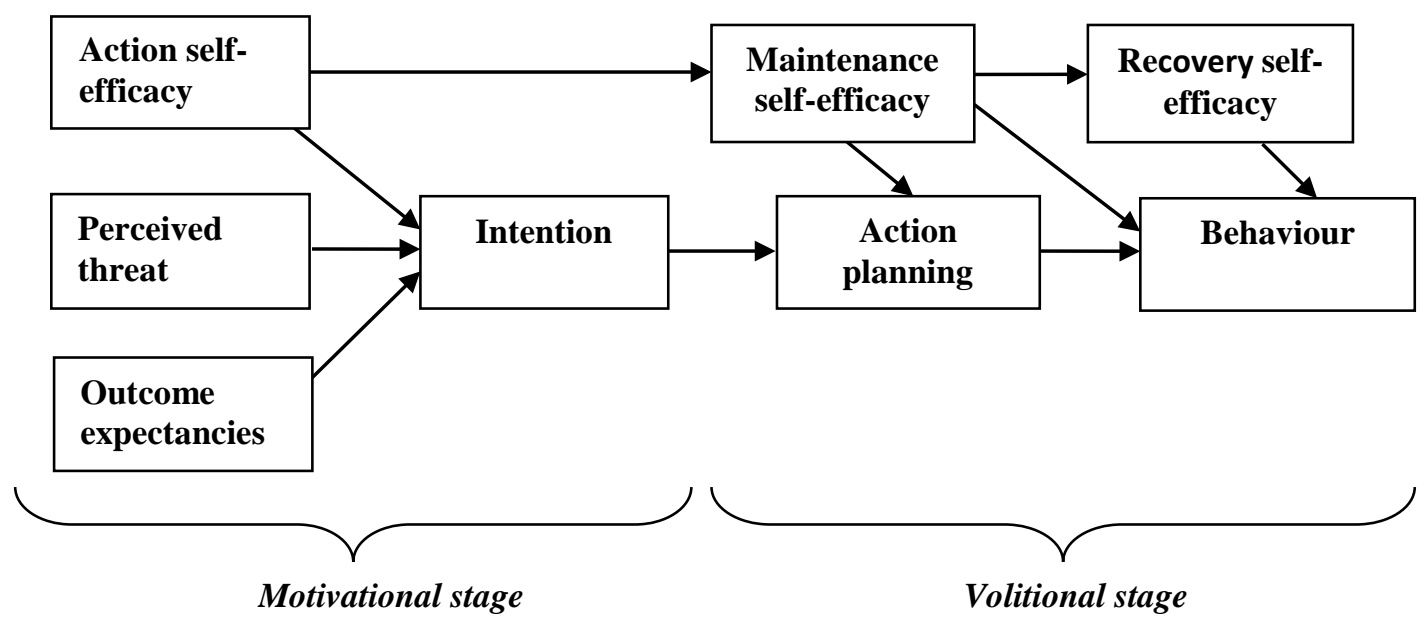


Figure 2

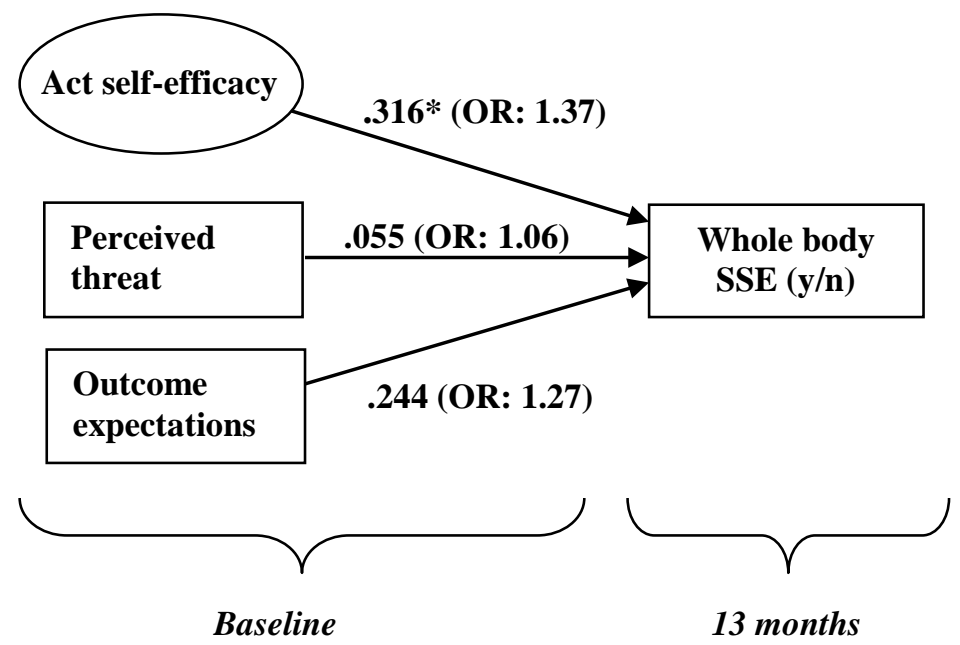

${ }^{*} \mathbf{p}<\mathbf{0 . 0 5}$ 
Figure 3
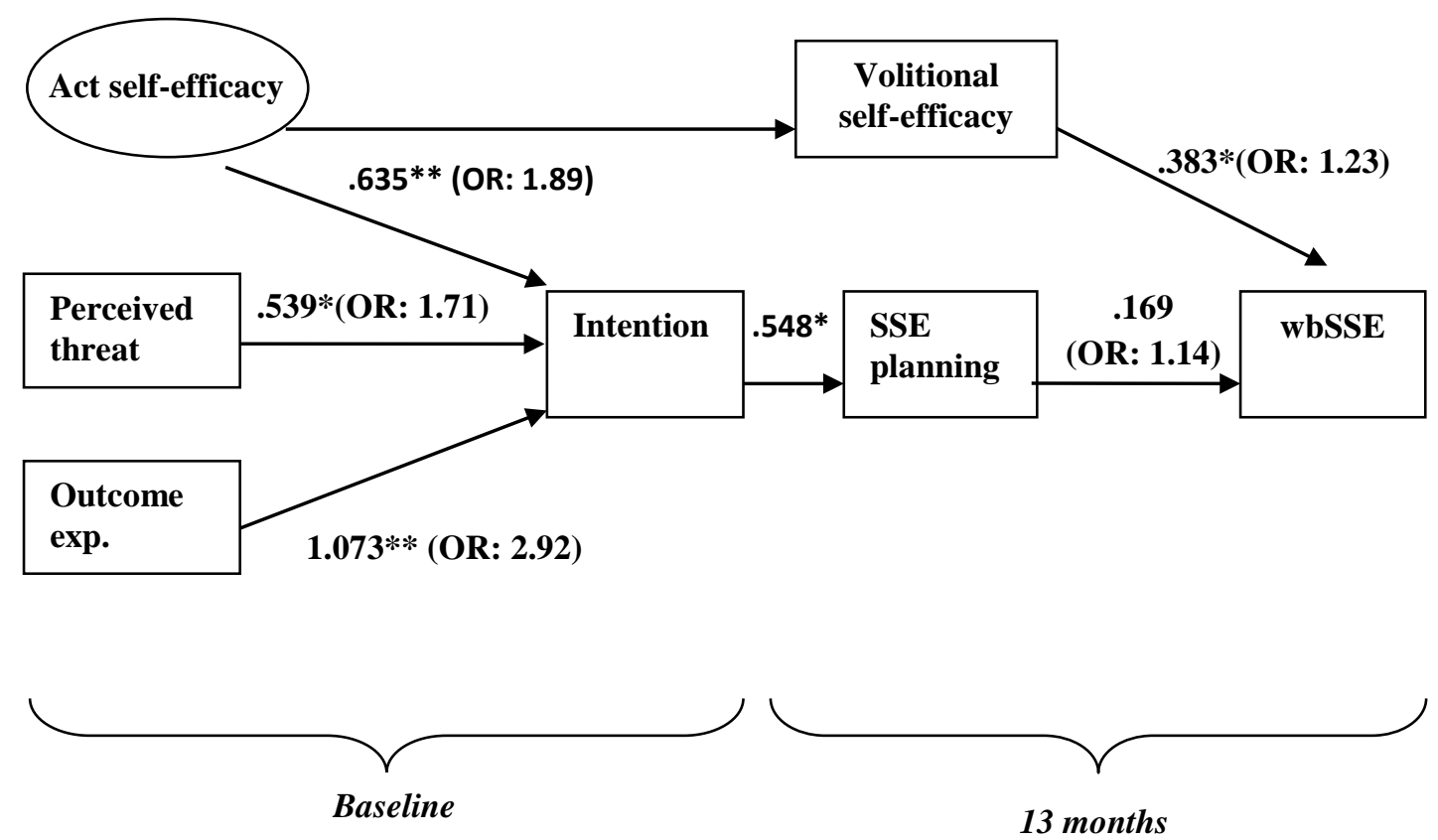

${ }^{*} \mathbf{p}<0.05 ; * * \mathbf{p}<0.001$ 
Figure 4

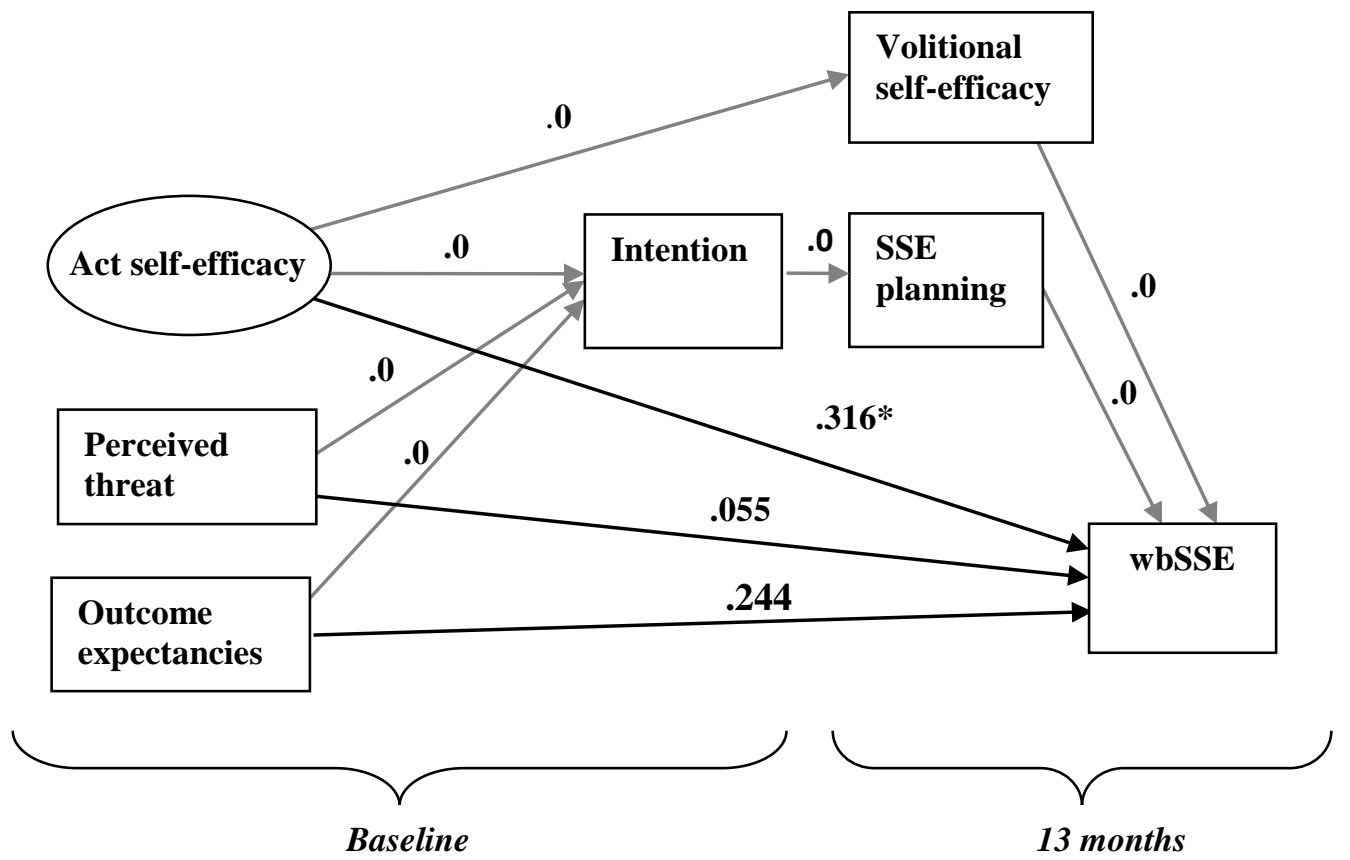

${ }^{*} \mathbf{p}<\mathbf{0 . 0 5} ;{ }^{* *} \mathbf{p}<\mathbf{0 . 0 0 1}$ 
Table 1: Participants' baseline demographic and health characteristics ${ }^{\text {a }}$

\begin{tabular}{lll}
\hline Characteristic & $\mathrm{n}=410$ & (\%) \\
\hline Area of Queensland & & \\
$\quad$ Urban & 194 & $(48.0)$ \\
$\quad$ Rural & 210 & $(52.0)$
\end{tabular}

Marital status

Does not live with a partner $\quad 58$

Married/lives with partner

351

Age group

50-60 years

$61-70$ years

71-90 years

179

Highest level of education completed

Less than junior high school $\quad 30$

Completed junior high school

Completed senior high school

68

Trade or technical certificate or diploma

University or college degree

85

Household income (yearly, before tax)

$\begin{array}{lcc}\text { Less than } \$ 20,000 & 48 \\ \$ 20,001 \text { to } \$ 40,000 & 96 & (11.7) \\ \$ 40,001 \text { to } \$ 60,000 & 76 & (18.5) \\ \$ 60,001 \text { to } \$ 80,000 & 44\end{array}$

Has had a Prostate Specific Antigen (PSA) test 
Table 2: Questionnaire items used to measure constructs of the HBM or HAPA

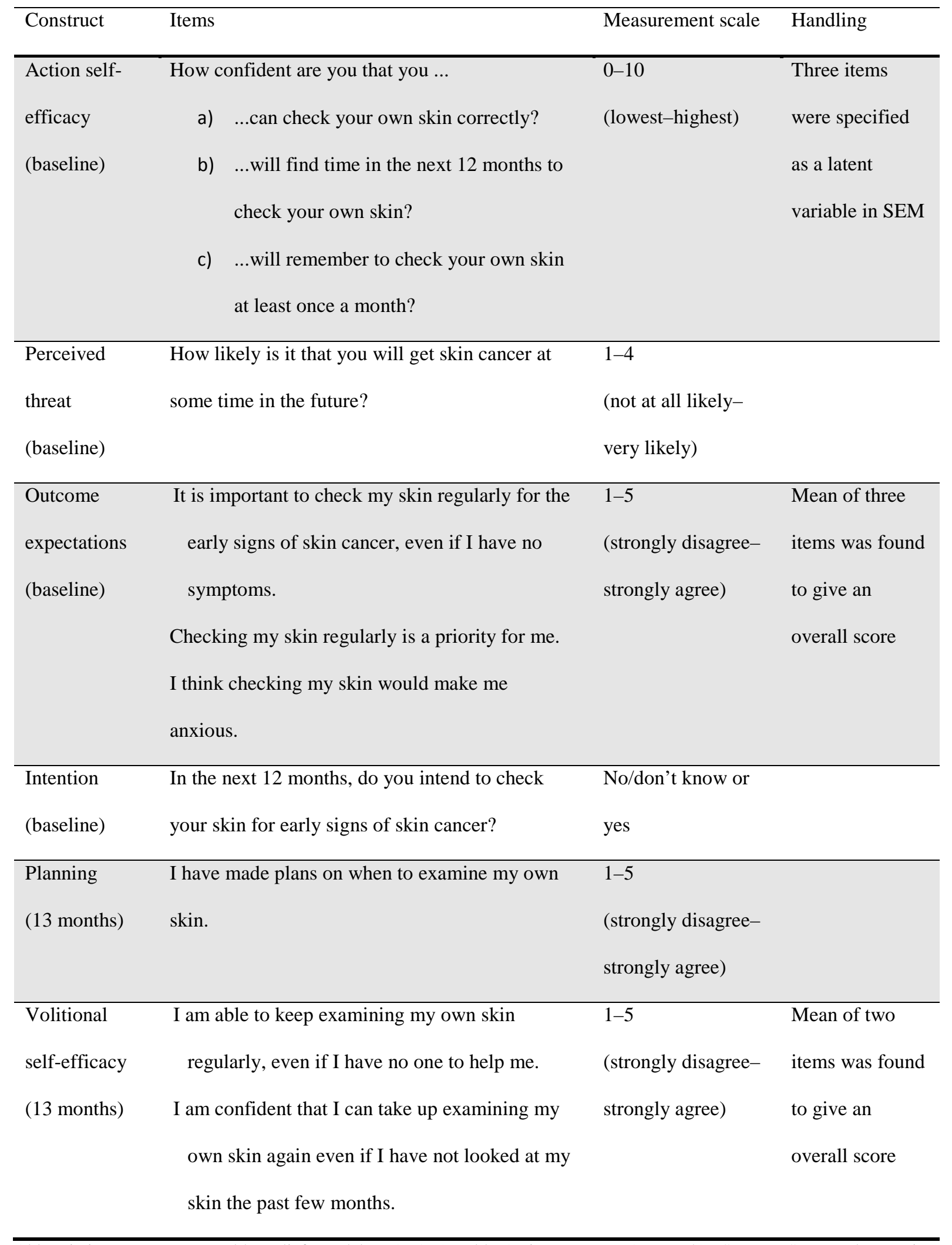

Abbreviations: HBM - Health Belief Model; HAPA (Health Action Process Approach); SEM - Structural Equation Modelling 
Table 3: Revised HBM and HAPA model comparison

\begin{tabular}{llll}
\hline Model & Loglikelihood & Number of free & AIC \\
& value & parameters & \\
\hline Null model (revised HBM): HAPA paths & -4401.59 & 17 & 8837.18 \\
constrained to equal zero & & & 8688.85 \\
Alternative model (HAPA): Unconstrained model & -4319.42 & 25 & \\
with all HAPA paths freely estimated, includes & & & \\
direct paths from baseline motivational factors to & & & \\
behaviour & & \\
\hline
\end{tabular}

\section{Formula applied:}

$D=-2 \times(\ln ($ likelihood for null model $)-\ln ($ likelihood for alternative model $))$

$D=-2 \times(-4401.59)-(-4319.42)$

$D=-2 \times-82.17$

$D=164.34$

Degrees of freedom $=25-17=8$

Abbreviations: HBM = Health Belief Model; HAPA Health Action Process Approach 